\title{
The Comparison of Iris Recognition using Principal Component Analysis, Log Gabor and Gabor Wavelets
}

\author{
Pravin S. Patil \\ North Maharashtra University \\ Umavinagar, Jalgaon \\ Maharashtra, India
}

\author{
S. R. Kolhe \\ North Maharashtra University \\ Umavinagar, Jalgaon \\ Maharashtra, India
}

\author{
R. V. Patil \\ BSD College of Engineering \\ Dhule, Mahrashtra \\ India
}

\author{
P. M. Patil \\ RMD Sinhghad Technical Institutes Campus \\ Warje , Pune \\ Maharashtra, India
}

\begin{abstract}
With an ever growing emphasis on security systems, automated personal identification based on biometrics has been getting extensive focus in both research and practical over the last decade. The methods for iris recognition mainly focus on feature representation and matching. As we known traditional iris recognition method is using Gabor Wavelet features, the iris recognition is performed by a 256 byte iris code, which is computed by applying Gabor wavelets to a given portion of iris. Log Gabor wavelet based features are invariant to changes in brightness and illumination whereas techniques like principal component analysis can produce spatially global features. The goal of this paper is to compare feature extraction algorithm based on PCA, Log Gabor Wavelet and Gabor Wavelet. We use these methods to generate feature vectors that could represent iris efficiently. Conclusions based on comparisons can provide useful information for further research. Performance of these algorithms is analyzed using CASIA database.
\end{abstract}

\section{General Terms}

Security, Biometrics, Iris Recognition

\section{Keywords}

PCA, Gabor Wavelet, Log Gabor Wavelet, Euclidean Distance, Hamming Distance.

\section{INTRODUCTION}

In recent years, accurate automatic personal identification is becoming more and more significant to the operation of security systems. Biometrics [10] employs physiological or behavioral characteristics to accurately identify each subject. Commonly used biometric features include face, fingerprints, voice, iris, retina, gait, palm-prints, hand geometry, dental radiographs etc. [10], [11]. Of all these Biometrics, fingerprint identification is one of the most famous and publicized biometrics and has been successfully used in security applications [10]. Face recognition and speaker recognition have also been widely studied over the last 30 years, whereas iris recognition is a newly emergent approach to personal identification in the last decade.

Among all biometrics (such as fingerprint, face, palmprint, gait, voice, iris, dental radiographs etc.), iris recognition is the most consistent one [11]. The iris is a thin circular diaphragm, which lies between the cornea and the lens of the human eye. The pattern of the human iris differs from person to person; there are not ever two irises alike, not even for genetically identical twins [1], [9], [10], [11]. The iris is considered one of the most stable biometric, as it is believed to not change significantly during a person's lifetime and its physiological response to light, which provides the detection of a dead or artificial iris, avoiding this kind of counterfeit [9], [10], [11]. Other properties of the human iris that increase its suitability for use in automatic identification include its inherent isolation and protection from the external environment, being an internal organ of the eye, behind the cornea and the aqueous humor.

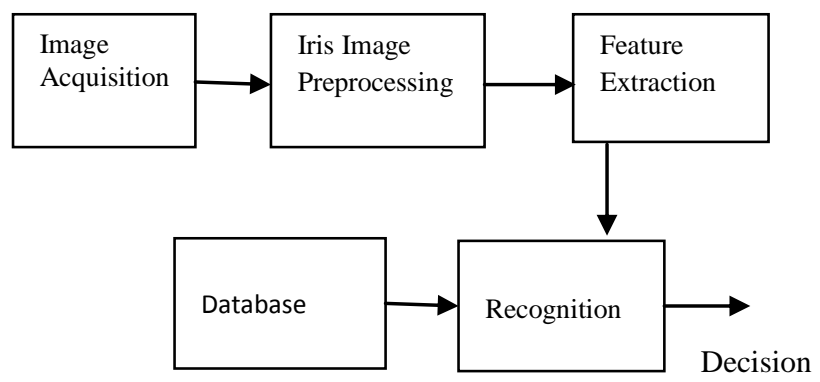

Figure 1.Typical Iris Recognition system

A typical iris recognition system is schematically shown in Fig. 1. The whole iris recognition process [1], [2], [4], [17] is basically divided into four steps: 1) Image acquisition; 2) iris image preprocessing; 3) iris feature extraction; and 4) matching. Nowadays, various algorithms for iris recognition have been presented. Furthermore, the preprocessing of iris image includes four aspects: localization, normalization, enhancement, denoising, and the selection of iris valid areas [7].

Various algorithms have been applied for feature extraction and pattern matching processes [7], [18], [22], [23], [32], [4]. These methods use local and global features of the iris [7]. A great deal of advancement in iris recognition has been made through these efforts; therefore, a detailed performance comparison of all these algorithms is necessary. The goal of this paper is to compare feature extraction algorithm based on PCA, Log Gabor Wavelet and Gabor Wavelet.

The remainder of this paper is organized as follows: Section 2 briefly discuss the image preprocessing including iris localization, iris normalization and iris enhancement. A detailed description of the feature extraction based on PCA, Log Gabor Wavelet and Gabor Wavelet is given in Section 3. Section 4 reports experiments and results. Section 5 concludes this paper. 


\section{IMAGE PREPROCESSING}

A captured iris image contains not only the region of interest (iris) but also some 'unuseful' parts (e.g. eyelid, pupil etc.). So, the image cannot be used directly without preprocessing. In addition, a change in the camera-to-face distance may result in the possible variation in the size of the same iris [1], [7]. Furthermore, the brightness is not uniformly distributed because of non-uniform illumination [19], [24]. For the purpose of recognition, the original image needs to be preprocessed to localize iris, normalize iris, and reduce the impact of the factors mentioned above. The preprocessing is described in the following subsections.

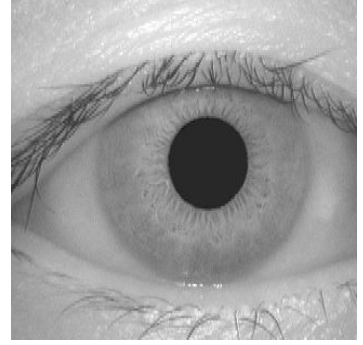

(a)

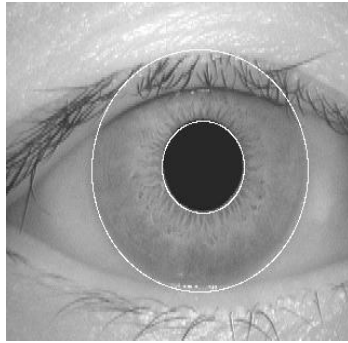

(b)
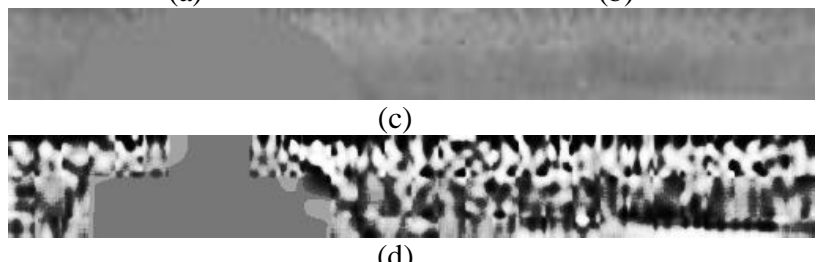

(d)

Figure 2. Preprocessing of Iris Image

(a) Original Iris Image (b) Image after Iris Localization

(c) Normalization of Iris Image (d) Enhanced iris image

\subsection{Iris Localization}

The iris localization is to identify the iris area between pupil and sclera from an eye image. Both the inner boundary and the outer boundary of a typical iris can be identified as circles [1], [7], [17], [19]. However, the two circles are usually not co-centric. The method we utilized for iris localization includes simple filtering, edge detection, and Hough transform. The overall method is very efficient and reliable [19]. An example of iris localization is shown in Figure $2 b$. We can see that the iris can be exactly localized using this technique.

\subsection{Iris Normalization}

A normalization process is needed to compensate for size variations due to the possible changes in the camera-to-face distance and to facilitate the feature extraction process by transforming the iris area represented by polar coordinate system into Cartesian coordinate system [2], [19]. Here, we anti-clockwise plot the iris ring to a rectangular block of texture of a fixed size. According to the requirement of feature extraction, this block is then divided into smaller subimages. The result after iris normalization is shown in Figure 2c.

\subsection{Iris Image Enhancement and Denoising}

The normalized iris image still has low contrast and may have non-uniform illumination caused by the position of light sources [1], [2], [7]. All these may affect subsequent feature extraction and pattern matching. We enhance the iris image by means of local histogram equalization and remove highfrequency noise by filtering the image with a low-pass
Gaussian filter. Figure 1d shows the preprocessing result of an iris image.

The iris has a particularly unique structure and provides rich texture information. So, it is necessary to explore representation methods which can describe global and local features in an iris. This section presents detailed description of iris recognition methods based on PCA, Gabor Wavelet and Log Gabor Wavelet.

\section{FEATURE EXTRACTION}

\subsection{Feature Extraction with Principal Component Analysis}

The aim of feature extraction is to find a transformation from an n-dimensional observation space to a smaller $\mathrm{m}$ dimensional feature space [29]. Main reason for performing feature extraction is to reduce the computational complexity for iris recognition. Most existing iris recognition methods are based on the local properties such as phase, shape, and so on [7]. However, iris image recognition based on local properties is difficult to implement. Principal component analysis can produce spatially global features [16], [17, [18]. Principal components analysis, or PCA (also called the KarhunenLoeve, or $\mathrm{K}$-L, method), searches for $k n$-dimensional orthogonal vectors that can best be used to represent the data, where $k \leq n$. The original data are thus projected onto a much smaller space, resulting in data reduction.

PCA was invented in 1901 by Karl Pearson [30]. Principal component analysis (PCA) is a classic technique used for compressing higher dimensional data sets to lower dimensional ones for data analysis, visualization, feature extraction, or data compression. PCA involves the calculation of the eigenvalue decomposition of a data covariance matrix or singular value decomposition of a data matrix, usually after mean centering the data for each attribute [30].

We employ this method to extract the iris regions features, and apply these steps [16], [18], [30].

Step 1: Get normalized data from the iris regions. 2-D iris image is represented as 1-D Vector by concatenating each row (or Column) into a long vector

Step 2: Subtract the mean image from each image vector.

Step 3: Calculate the covariance matrix.

Step 4: Calculate the eigenvectors and eigenvalues of the covariance matrix.

Step 5: The eigenvectors are sorted from high to low according to their corresponding eigenvalues. Choose components and forming a feature vector

Step 6: Deriving the new data set

Once we have chosen the components, we simply take the transpose of the vector and multiply it on the left of the original data set, transposed.

\section{FinalDataSet $=$ RowFeatureVector $\mathrm{x}$ RowMeanAdjust}

Where RowFeatureVector is the matrix with the eigenvectors in the columns transposed so that the eigenvectors are now in the rows, with the most significant eigenvector at the top, and RowMeanAdjust is the mean adjusted data transposed. The data items are in each column, with each row holding a separate dimension [30]. 


\subsection{Feature Extraction with Gabor}

\section{Wavelet}

John G. Daugman, a Professor of Cambridge University [1], [2], [3] proposed a typical and successful iris recognition system that makes use of a decomposition derived from application of two dimensional version of Gabor filters to the image data to extract its phase information. Decomposition of a signal is accomplished using a quadrature pair of Gabor filters, with a real part specified by a cosine modulated by a Gaussian, and an imaginary part specified by a sine modulated by a Gaussian [13]. The real and imaginary filters are also known as the even symmetric and odd symmetric components respectively. The centre frequency of the filter is specified by the frequency of the sine/cosine wave, and the bandwidth of the filter is specified by the width of the Gaussian. A 2D Gabor filter over the an image domain $(x, y)$ is represented as

$G(x, y)=e^{-\pi\left[\left(x-x_{0}\right)^{2} / \alpha^{2}+\left(y-y_{0}\right)^{2} / \beta^{2}\right]} e^{-2 \pi i\left[u_{0}\left(x-x_{0}\right)+v_{0}\left(y-y_{0}\right)\right]}(1)$

Where $\left(x_{0}, y_{0}\right)$ position in the image, $(\alpha, \beta)$ specify effective width, and length and $\left(u_{0}, v_{0}\right)$ specify modulation which has spatial frequency $\omega_{0}=\sqrt{u_{0}^{2}+v_{0}^{2}}$.

Daugman[1], [2], [3] demodulates the output of the Gabor filters in order to compress the data. This is done by quantizing the phase information into four levels, for each possible quadrant in the complex plane. It has been shown [13] that phase information, rather than amplitude information provides the most important information within an image. Taking only the phase will allow encoding of discriminating information in the iris, while discarding redundant information such as illumination, which is represented by the amplitude component.

These four levels are represented using two bits of data, so each pixel in the normalized iris pattern corresponds to two bits of data in the iris template. A total of 2,048 bits are calculated for the template, and an equal number of masking bits are generated in order to mask out corrupted regions within the iris. This creates a compact 256-byte template, which allows for efficient storage and comparison of irises. The Daugman system [1], [2], [3] makes use of polar coordinates for normalization, therefore in polar form the filters are given as

$H(r, \theta)=e^{-i \omega\left(\theta-\theta_{0}\right)} e^{-\left(r-r_{0}\right)^{2} / \alpha^{2}} e^{-\left(\theta-\theta_{0}\right)^{2} / \beta^{2}}$

Where $(\alpha, \beta)$ are same as in equation (1), and $\left(r_{0}, \theta_{0}\right)$ specify centre frequency of the filter.

The demodulation and phase Quantization process can be represented as

$h_{\{R e, I m\}}=$

sgnRe,Im $|\oint| \varnothing I p, \varnothing e-i \omega \theta 0-\varnothing e-r 0-p 2 e-\theta 0-\varnothing 2 p d p d \emptyset$

where $h_{\{R e, I m\}}$ can be regarded as a complex valued bit whose real and imaginary components are dependent on the sign of the $2 \mathrm{D}$ integral, and $\mathrm{I}(\mathrm{p}, \emptyset)$ is the raw iris image in a dimensionless polar coordinate system. For a detailed study of 2D Gabor wavelets see [13].

\subsection{Feature Extraction with Log Gabor Wavelet}

A disadvantage of the Gabor filter is that the even symmetric filter will have a DC component whenever the bandwidth is larger than one octave. However, Log Gabor can cover large frequency space while still maintaining a zero DC component in the even symmetric filter [24], [25], [27]. Therefore the background brightness will not affect the extraction of the pure phase information of iris texture. Filters are constructed in the frequency domain using a polar co-ordinate system [24]. On the linear frequency scale, log Gabor has a transfer function of the form

$$
g(f)=e^{\frac{-\left(\log \left(f / f_{0}\right)\right)^{2}}{2\left(\log \left(\sigma / f_{0}\right)\right)^{2}}}
$$

Where $f_{0}$ is the centre frequency of filter and $\sigma$ is the bandwidth of filter.

If we let $\mathrm{I}(\mathrm{x}, \mathrm{y})$ denote the image and $W_{n}^{e}$ and $W_{n}^{o}$ denote the even symmetric (cosine) and odd-symmetric (sine) wavelets at a scale n, we can think of the responses of each quadrature pair of filters as forming a response vector,

$$
[e n(x, y), \text { on }(x, y)]=\left[I(x, y) * W_{n}^{e}, I(x, y) * W_{n}^{o}\right]
$$

The amplitude of the transform at a given wavelet scale is given by

$$
A_{n}(x, y)=\sqrt{e_{n}(x, y)^{2}+O_{n}(x, y)^{2}}
$$

and the phase is given by

$$
\phi_{n}=a \tan 2\left(e_{n}(x, y), o_{n}(x, y)\right)
$$

Where $\mathrm{A}_{\mathrm{n}}(\mathrm{x}, \mathrm{y})$ is the amplitude and $\phi_{\mathrm{n}}$ is the phase of the angle. During the phase extraction, the iris image is divided into $\mathrm{m}$ by $\mathrm{n}$ blocks. Consequently, information in each block is encoded into 2-bit codes. Thus the phase information in each block is described by 2-bit codes and totally $2 \mathrm{mn}$ bits to describe the whole iris.

\section{EXPRIMENTAL RESULTS AND COMPARISION}

Evaluating the performance of biometric algorithms is a difficult issue. Currently, there is also no detailed comparison among the iris recognition methods [7]. For the purpose of comparison; we implement these methods according to the published papers. To compare their performance, we construct an iris image database named CASIA Iris Database. We use images of eyes from 30 persons, and every person has 10 images of eyes. MATLAB image processing tools were used to implement system. We use the usual methods to locate and normalize iris regions, and use the three methods mentioned above to extract the feature. Therefore, we only analyze and compare the accuracy and computational complexity of feature representation and matching of these methods. For each iris pattern, we randomly choose several samples for training and the rest for testing. After feature extraction, an iris image is represented as a feature vector. We used Euclidean Distance and Hamming Distance similarity measures to measure the similarity of iris features. Two distance measure lead to similar results and recognition result does not vary drastically. Recognition rate is shown in Table 1

Table 1. Experimental Results

\begin{tabular}{|c|c|c|c|}
\hline Method & $\begin{array}{c}\text { Feature } \\
\text { Vector } \\
\text { Length (Bits) }\end{array}$ & Classifier & Recognition Rate \\
\hline $\begin{array}{c}\text { Gabor } \\
\text { Wavelet }\end{array}$ & 2048 & $\begin{array}{c}\text { Hamming } \\
\text { Distance }\end{array}$ & $99 \%$ \\
\hline $\begin{array}{c}\text { Log Gabor } \\
\text { Wavelet }\end{array}$ & 1024 & $\begin{array}{c}\text { Hamming } \\
\text { Distance }\end{array}$ & $92.4 \%$ \\
\hline PCA & 1100 & $\begin{array}{c}\text { Euclidean } \\
\text { Distance }\end{array}$ & $90.2 \%$ \\
\hline
\end{tabular}




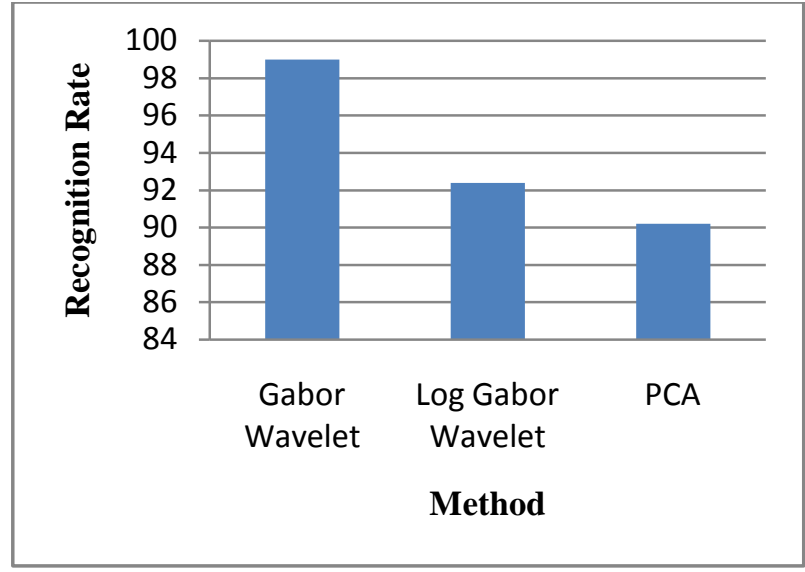

Fig. 3. Recognition Rate of Gabor Wavelet, Log Gabor Wavelet and PCA

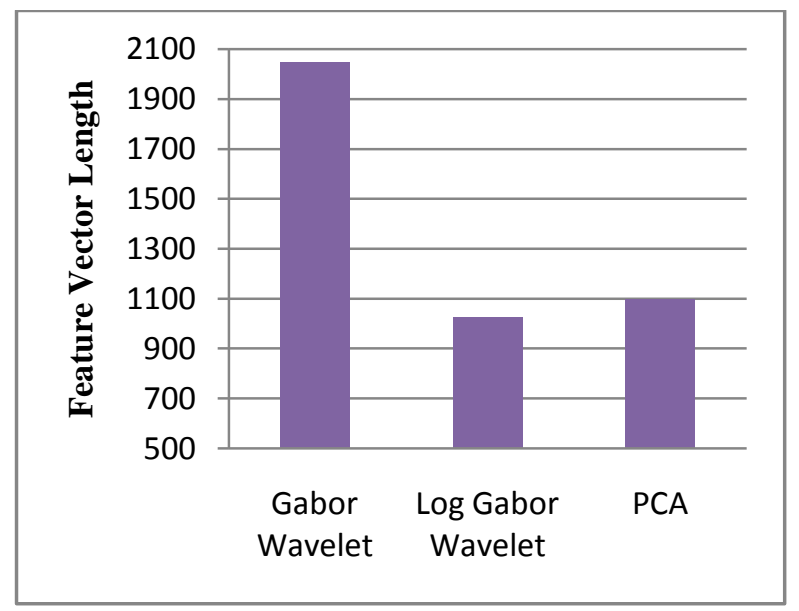

Fig. 4. Feature Vector Length of Gabor Wavelet, Log Gabor Wavelet and PCA

The results clearly demonstrate the effectiveness of the Daugman's method. With strict image quality control, Gabor filter based method is slightly better than the Log Gabor wavelet and PCA based methods. Gabor wavelet based method captures much more information in much smaller local regions, which makes this method better than others [7]. However, the iris capture devices in use are mostly exposed to the natural scene, so the natural illumination or other variant conditions sometimes can greatly influence the iris images captured and further impact the recognition result. Out of this consideration, an iris feature extraction method based on Log Gabor Wavelet can tolerant to illumination variations.

\section{CONCLUSION}

From the experimental results mentioned above, it has been proved that Daugman's method based of Gabor wavelet is effective and has highest recognition rate under strict image quality control. However iris images have low contrast and may have non-uniform illumination caused by the position of light sources. Log Gabor Wavelet is invariant to changes in image brightness or contrast and suitable to represent features under varying conditions. But However Gabor wavelet and Log Gabor Wavelet methods have high computational complexity compared to PCA based method. PCA based method is computationally inexpensive and requires less time and therefore we may consider the algorithm of PCA if the noise is not strong.

\section{REFERENCES}

[1] J. G. Daugman (1993), "High confidence visual recognition of persons by a test of statistical independence", IEEE Transactions on Pattern Analysis and Machine Intelligence, 15(11), 1148-1161.

[2] J. Daugman (1994), "Biometric Personal identification System based on iris analysis", US patent no. 529160.

[3] J. Daugman (2003), "Demodulation by Complex valued wavelets for stochastic pattern recognition", International Journal of Wavelets, Multiresolution and Information Processing, 1(1), 1-17

[4] W. W. Boles and B. Boashash (1998), "A human identification technique using images of the iris and wavelet transform", IEEE Transactions on Signal Processing, 46(4), 1185-1188.

[5] J. Daugman (2001), "Statistical Richness of Visual Phase Information: Update on Recognizing Persons by Iris Patterns," International Journal of Computer Vision, 45(1), 25-38.

[6] Hao Mang, Cuiping Xu (2006), "Iris Recognition Algorithms based on Gabor Wavelet Transforms", Proceedings of the 2006 IEEE International Conference on Mechatronics and Automation, Luoyang, China, 1785-1789

[7] L.Ma, T. Tan, Y.Wang, and D. Zhang (2003), "Personal identification based on iris texture analysis," IEEE Transactions on Pattern Analysis and Machine Intelligence, 25(12), 1519-1533.

[8] L.Ma, T. Tan, Y. Wang, and D. Zhang (2004), "Efficient iris recognition by characterizing key local variations," IEEE Transactions on Image Processing, 13(6), 739-750.

[9] R. Johnson (1991), "Can Iris Patterns Be Used to Identify People?", Chemical and Laser Sciences Division LA-12331-PR, Los Alamos Nat'l Laboratory, Calif.

[10] Jain, R. Bolle and S. Pankanti (1999) ," Biometrics: Personal Identification in a Networked Society", Kluwer Academic Publishers.

[11] A. Mansfield and J. Wayman (2002), "Best Practice Standards for Testing and Reporting on Biometric Device Performance," Nat'l Physical Laboratory of UK.

[12] L. Ma, Y. Wang, and T. Tan (2002), "Iris Recognition Based on Multichannel Gabor Filtering," Proc. Fifth Asian Conf. Computer Vision, 279-283.

[13] T. Lee (1996), "Image Representation Using 2D Gabor Wavelets," IEEE Trans. Pattern Analysis and Machine Intelligence, 18(10), 959-971.

[14] P. Belhumeur, J. Hespanha, and D. Kriegman (1997), "Eigenfaces vs. Fisherfaces: Recognition Using Class Specific Linear Projection," IEEE Trans. Pattern Analysis and Machine Intelligence, 19(7), 711-720.

[15] J. Zhang and T. Tan (2002), "Brief Review of Invariant Texture Analysis Methods," Pattern Recognition, 35(3), 735-747.

[16] J. Beveridge, K. She, B. Draper, and G. Givens (2001), "A Nonparametric Statistical Comparison of Principal Component and Linear Discriminant Subspaces for Face Recognition," Proc. IEEE Conf. Computer Vision and Pattern Recognition, 535-542.

[17] L. Masek (2003) "Recognition of human iris patterns for biometrics identification", B. Eng. thesis, University of Western Australia, Perth, Australia.

[18] V. Dorairaj and N. Schmid, and G. Fahmy (2005), "Performance Evaluation of Iris Based Recognition System Implementing PCA and ICA Techniques," in Proc. of the SPIE 2005 Symp. on Defense and Security, Conf. 5779, Orlando,.

[19] Peng Fei Zhang, De-Sheng Li, Qi Wang (2004), "A Novel Iris Recognition Based On Feature Fusion", Proceedings of the Third International Conference on Machine Learning and Cybernetics, Shanghai, 36613665 . 
[20] "CASIA Iris http://www.sinobiometrics.com

Image

Database,"

11] J. Cui, Y. Wang, J. Huang, T. Tan, and Z. Sun (2004), "An Iris Image Synthesis Method Based on PCA and Super-Resolution," Proc. 17 $7^{\text {th }}$ Int'l Conf. Pattern Recognition, 471-474.

[22] Y. Huang, S. Luo, and E. Chen (2002), "An Efficient Iris Recognition System," Proc. Int'l Conf. Machine Learning and Cybernetics, 450-454.

[23] Peng Yao, Jun Li, Xueyi Ye, Zhenquan Zhuang, Bin Li (2006), "Iris Recognition Algorithm using modified Log Gabor Filters", The 18th International Conference on Pattern Recognition(ICPR'06), IEEE Computer Society, 461-464.

[24] P Kovesi. (1999), "Image features from phase congruency.", Videre Journal of Computer Vision Research, 1(3), 1-27.

[25] P. Kovesi. (2003)," Phase congruency detects corners and edges.",In DICTA, Sydney.

[26] Ety Navon, Often Miller, Amir Averabuch (2005), "Color image segmentation based on adaptive local thresholds", Image and vision computing, 69-85.

[27] M. C. Morrone, Owens (1987), "Feature Detection From Local Energy", Pattern Recognition Letters, 310-313.
[28] Zheng Liu, R. Leganneire (2006), "On the Use of Phase Congruency to evaluate Image Similarity", IEEE International Conference on Accoustics, Speech and Signal Processing, 937-940.

[29] B. J. Lei, Emile A. Hendriks, M.J.T. Reinders, "On Feature Extraction from Images", Technical Report, MCCWS Project, Information and Communication Theory Group, Tudeft.

[30] Jin-Xin Shi and Xiao-Feng Gu (2010)," The Comparision of Iris Recongnition Using Prinncipal Component Analysis, Independent Component Analysis and Gabor Wavelets", 3rd IEEE International Conference on Computer Science and Information Technology (ICCSIT), 61-64.

[31] R. O. Duda, P. E. Hurt (1972), “ Use of Hough transform to detect line and curves in pictures", Communication of ACM.

[32] D.M. Monro and D. Zhang (2005), "An Effective Human Iris Code with Low Complexity," Proc. IEEE Int'l Conf. Image Processing, 3(3), 277-280.

[33] A.K. Jain, A. Ross, and S. Prabhakar (2004), "An Introduction to Biometric Recognition," IEEE Trans. Circuits and Systems for VideoTechnology, 14, 4-20. 